\title{
Oral electro galvanism
}

\section{Fatma Frikha, Emna Bahloul, Hela Mesrati, Meriem Amouri, Hamida Turki}

Département de Dermatologie Vénérologie, CHU Hédi Chaker, Sfax, Tunisie

Corresponding author: Dr. Fatma Frikha, E-mail: frikhafat@gmail.com

\begin{abstract}
Lesions which are clinically and/or histopathologically compatible with a diagnosis of lichen planus seem to develop as readily when electrical microcurrents are present as do lesions that are compatible with a diagnosis of leukoplakia. We report a case of oral electro galvanism in a 60-year-old woman. These microcurrents are the result of the simultaneous use of dissimilar metals (precious metals, stainless-steel alloys, amalgam) in dentistry. The role of electrogalvanic microcurrents in the development of white lesions of the oral mucosa is substantiated by the disappearance or regression of lesions after change of the different metals.
\end{abstract}

Key words: Dental materials; Lichen planus; Galvanism 


\title{
Electrogalvanisme oral
}

\section{Fatma Frikha, Emna Bahloul, Hela Mesrati, Meriem Amouri, Hamida Turki}

\author{
Département de Dermatologie Vénérologie, CHU Hédi Chaker, Sfax, Tunisie
}

Corresponding author: Dr. Fatma Frikha, E-mail: frikhafat@gmail.com

\begin{abstract}
RÉSUMÉ
Les lésions qui sont cliniquement et/ou histologiquement compatibles avec le diagnostic de lichen plan semblent se développer aussi facilement en présence de micro-courants électriques, comme pour les lésions de leucoplasie. Nous rapportons un cas d'électro-galvanisme oral chez une femme de 60 ans. Ces micro-courants sont le résultat de l'utilisation simultanée de métaux différents (métaux précieux, alliages d'acier inoxydable, amalgame) en dentisterie. Le rôle des micro-courants galvaniques dans le développement des lésions blanches de la muqueuse buccale est appuyé par la disparition ou la régression des lésions après le changement des différents métaux.
\end{abstract}

Mots clés: Matériaux dentaires; Lichen plan; Galvanisme

\section{INTRODUCTION}

En odontologie, différents alliages métalliques sont utilisés pour les prothèses, les restaurations et les dispositifs d'orthodontie. Ils peuvent être responsables de lésions au niveau de la muqueuse buccale par le biais d'un phénomène allergique de contact ou par électrogalvanisme (EG). L'EG est un micro-courant à basse tension crée par l'inter-réaction des différents métaux coexistant sous l'effet de la salive. Nous rapportons une observation de lichen buccal induit par un EG.

\section{OBSERVATION}

Il s'agit d'une femme âgée de 60 ans, sans antécédents pathologiques particuliers, qui consultait pour une stomatite douloureuse avec gêne alimentaire évoluant depuis quelques semaines résistantes à des soins locaux. A l'examen, il y avait des lésions blanchâtres non infiltrées linéaires siégeant au niveau de la face interne de la lèvre supérieure et de la muqueuse vestibulaire antéro-supérieure (Figs. la et $1 \mathrm{~b}$ ). Par ailleurs, le reste de l'examen était sans anomalies. La patiente n'était ni tabagique ni alcoolique et elle niait toute prise médicamenteuse. Le bilan hépatique ainsi que les sérologies des hépatites étaient sans anomalies. A la reprise de l'interrogatoire, la patiente déclarait l'apparition de sa gêne quelques jours après avoir fait des soins dentaires. A l'examen de sa dentition, elle avait des couronnes dentaires nouvelles (arcade dentaire supérieure gauche) et des amalgames usés et mis à nu (arcade dentaire supérieure droite) (Figs. 2 et 3), et vu que les lésions lichénoïdes étaient à distance de ces matériaux, nous avons évoqué le diagnostic d'un EG oral. En fait, les métaux utilisés étaient: inox avec un alliage cobalt chrome (côté gauche) et des amalgames en argent, cuivre, étain et mercure (côté droit), d'où l'effet d'une pile électrique. Les lésions lichénoïdes siégeaient ainsi sur le trajet du micro-courant. Etant donné que notre patiente était gênée et résistante aux soins locaux, nous avons procédé à une extraction des résidus d'amalgames remplacés par des implants et des couronnes en céramique du côté droit. Lévolution était bonne au bout de quelques mois (disparition de la gêne alimentaire et des lésions muqueuses).

\section{DISCUSSION}

LEG est un phénomène peu connu et largement sousestimé. Il requiert la présence de 3 conditions: 2 métaux de natures différentes, au potentiel d'oxydoréduction différent, la mise en contact électrique de ces 2 métaux et la présence d'un électrolyte les recouvrant (Salive) [1]. Certains métaux sont plus réactifs que

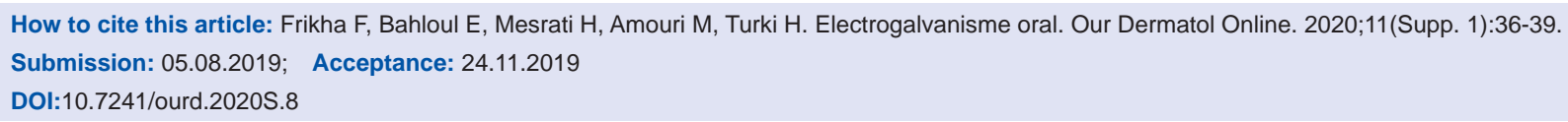




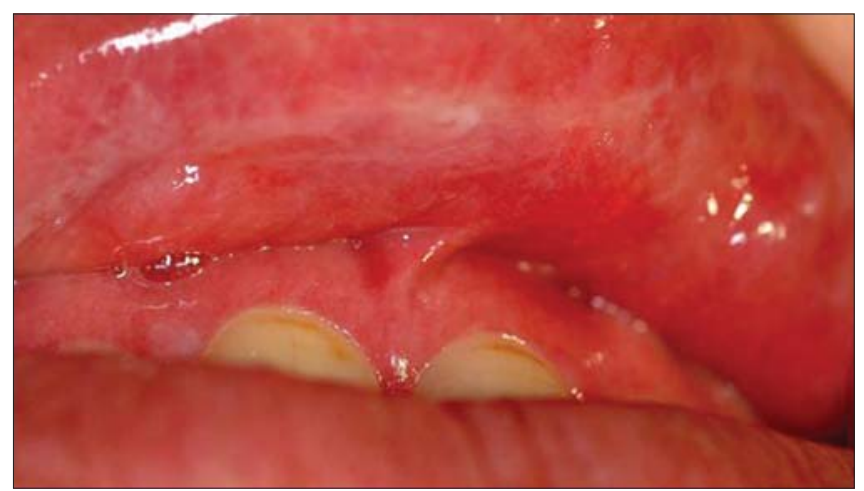

Figura 1a: Lésion blanchâtre linéaire non infiltrée au niveau de la face interne de la lèvre supérieure et de la muqueuse vestibulaire antéro-supérieure.

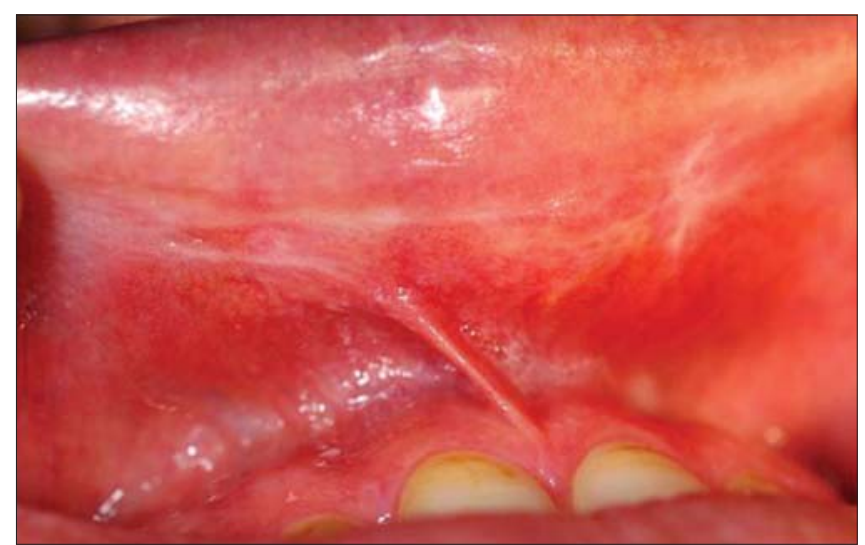

Figura 1b: Lésion blanchâtre linéaire non infiltrée au niveau de la face interne de la lèvre supérieure et de la muqueuse vestibulaire antéro-supérieure.

d'autres. Le métal le plus réactif passe en solution sous forme d'ions en générant un faible courant électrique. Lor qui est le métal le moins réactif, résiste à la corrosion, mais il provoque la corrosion de tout autre métal moins noble. C'est pourquoi, il faut absolument éviter d'associer des métaux précieux et non précieux dans une même bouche [2-4].

Les conséquences de l'EG peuvent être buccales et/ou à distance (ingestion et diffusion systémique des métaux corrodés). Des études récentes ont suggéré son rôle étiologique dans le développement de la leucoplasie orale et du lichen buccal [1]. Le traitement est symptomatique et surtout étiologique. La méthode de choix en cas de lésions lichénoïdes reste l'élimination de différents métaux en plaçant des obturations composites et des matériaux non métalliques. Cependant, dans certains cas les lésions de la muqueuse buccale deviennent irréversibles avec un risque de transformation maligne non négligeable, nécessitant une surveillance continue [5].

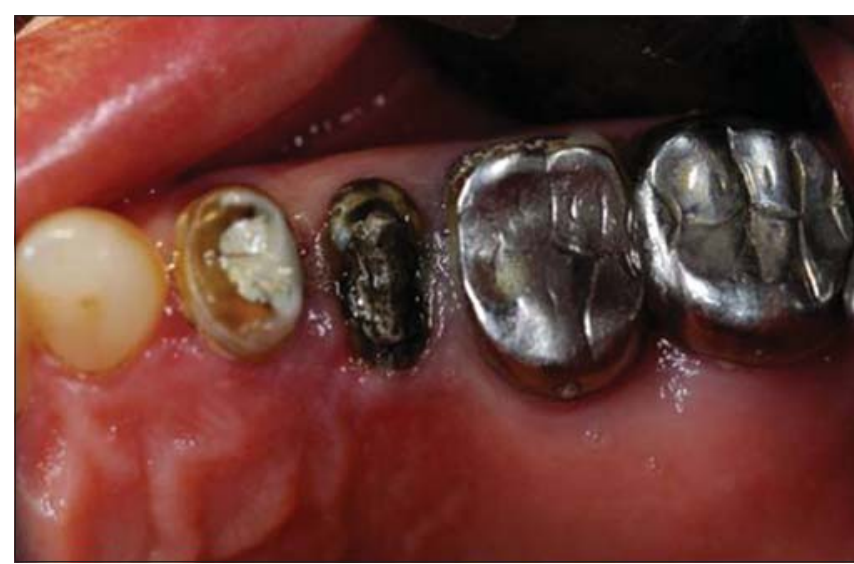

Figura 2: Couronnes dentaires (arcade dentaire supérieure gauche).

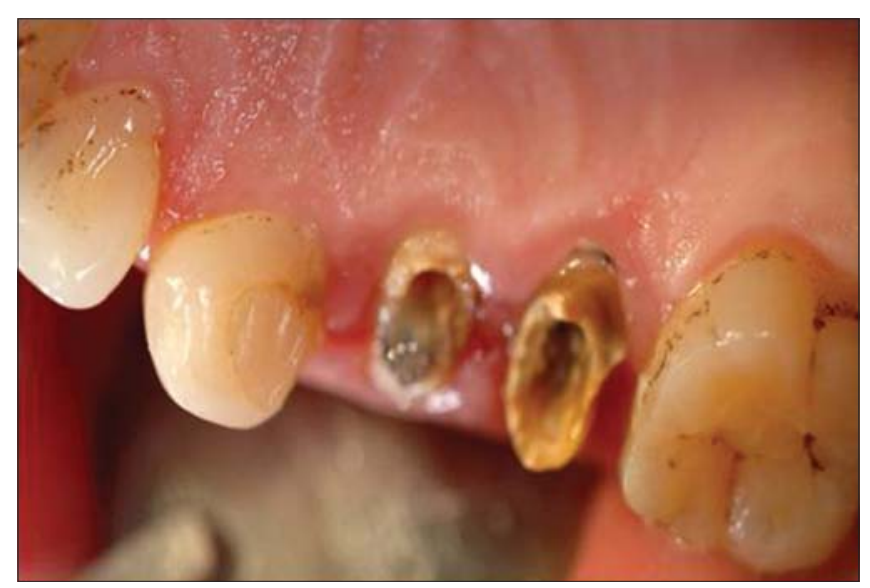

Figura 3: Amalgames usés et mis à nu (arcade dentaire supérieure droite).

\section{CONCLUSION}

Il existe une association évidente entre le lichen buccal, la corrosion des amalgames, la présence du bimétallisme et la présence d’or dans la bouche. Il faut éviter l'utilisation de restaurations métalliques différentes chez le même patient et privilégier des matériaux non métalliques tels que la résine composite, la céramique et la zircone.

\section{Consent}

The examination of the patient was conducted according to the Declaration of Helsinki principles.

The authors certify that they have obtained all appropriate patient consent forms. In the form the patient(s) has/have given his/her/ their consent for his/her/their images and other clinical information to be reported in the journal. The patients understand that their names and initials will not be published and due efforts will be made to conceal their identity, but anonymity cannot be guaranteed. 
www.odermatol.com

\section{REFERENCES}

1. Korraah A, Odenthal M, Kopp M, Vigneswaran N, Sacks PG, Dienes HP, et al. Induction of apoptosis and up-regulation of cellular proliferation in oral leukoplakia cell lines inside electric field. Oral Surg Oral Med Oral Pathol Oral Radiol. 2012;113:644-54.

2. Janos Inovay JB. Etiology of chronic diseases of the oral mucosa. 1960;884-90.

3. Stoopler ET, Charmelo-Silva S, Bindakhil M, Alawi F, Sollecito TP. Oral Lichen Planus Pemphigoides: Three Cases of a Rare Entity. Am J Dermatopathol. 2020;42:467-69.
4. Tamer F, Avci E. Decreased serum ferritin and vitamin D levels in patients with recurrent aphthous stomatitis. Our Dermatol Online. 2019;10:229-33.

5. Bdndczy J, Roed-petersen B. Clinical and histologic studies on electrogalvanically induced oral white lesions. 1979;319-23.

Copyright by Fatma Frikha, et al. This is an open-access article distributed under the terms of the Creative Commons Attribution License, which permits unrestricted use, distribution, and reproduction in any medium, provided the original author and source are credited.

Source of Support: Nil, Conflict of Interest: None declared. 